Die EU-Osterweiterung stellt zugleich eine wesentliche Herausforderung für die Reformfähigkeit der EU dar. Dabei dürfte die Schönwetterperiode für die (erweiterte) EU vorüber sein. Dies gilt möglicherweise auch für die Umweltpolitik, die bei der Süderweiterung durch unterstïtzende Strukturfonds abgesichert werden konnte und bei der Norderweiterung durch die umweltpolitisch fortgeschrittenen Staaten eher gestïtzt wurde. Aus den neuen Beitrittstaaten erscheinen aktuell weder umweltpolitische Initiativen erwartbar, noch wird ihnen umfassende finanzielle Unterstïtzung gewährt werden. Eine solche wäre jedoch angesichts der gesamteuropäischen ökologischen Vorteile durch die Harmonisierung, der induzierten ökonomischen Belastungen der MOEL sowie der ökonomischen Vorteile insbesondere einiger Staaten der EU, für die der Beitritt einem Förderprogramm der Umwelttechnikindustrie gleichkommt, zumindest teilweise angemessen. Ungeachtet dieser Einschränkungen stellen die Beitrittsverhandlungen für die MOEL einen wesentlichen Anreiz dar, trotz der vielfältigen anderen Transformationsprobleme Umweltaspekte nicht zu vernachlässigen.

\section{Anmerkungen}

(1) Vgl. Europäische Kommission: Agenda 2000, 1997.

(2) Vgl. für eine ausführliche Erörterung Ulrich Petschow/ Ralf Nordbeck/ Jan Nill: Der Prozeß der EU-Osterweiterung aus umweltpolitischer Perspektive. IÖW-Schriftenreihe 137/98 (im Erscheinen).

(3) Vgl. De Nocker, Leo et al.: Compliance Costing for Approximation of EU Environmental Legislation in the CEEC. Report to DG XI, Brüssel, European Commission, 1997.

(4) Vgl. Regional Environmental Center: Approximation of European Union Environmental Legislation. Budapest 1996.

(5) Vgl. hierzu bereits Stein, Regine: Westliche Hilfsprogramme für die Länder Mittel- und Osteuropas im Umweltsektor - Note: ungenügend. In: IÖW/VÖW-Informationsdienst, Nr. 2/1994, S.16-17.

(6) $\mathrm{Vgl}$ z.B. Caddy, J.: Harmonisation and asymmetry: environmental policy c0-ordination between the European Union and Central Europe. In: Journal of European Public Policy. Vol. 4 (1997), Nr. 3.

\section{Die Autoren}

Jan Nill und Ulrich Petschow sind Mitarbeiter des Forschungsfelds Umweltökonomie und -politik des IÖW. Kontakt: I0̈W, Giesebrechtstr. 13, 10629 Berlin, Tel. 030/ 884594-0, Fax 030/ 8825439, E-mail: Jan.Nill@ioew.de

Verbändevereinbarung: Instrument zur Behinderung des Wettbewerbs mit sauberem Strom

\title{
Diskriminierende Praxis
}

\author{
Die deutsche Energiewirtschaft steht vor einem Umbruch: Dort wo jahrzehnte- \\ lang Monopole das Sagen hatten, soll umfassender Wettbewerb einkehren. Das \\ neve Energierecht (1) ermöglicht den Verbrauchern im Prinzip zu wählen, wel- \\ chen Strom sie in Zukunft einkaufen wollen. Abgesehen von seinen umwelt- \\ und klimapolitischen Defiziten wird das neue Recht vor allem wegen seiner \\ wettbewerbsverzerrenden Elemente kritisiert.
}

$\mathrm{K}$ ernpunkt des angestrebten europäischen Stromhandels ist der Netzzugang aller Anbieter und Abnehmer (Verbraucher). Sowohl in der EU-Stromrichtlinie von 1997 als auch im neuen deutschen Energierecht ist der diskriminierungsfreie Netzzugang gesetzlich festgeschrieben. Dennoch ist absehbar, daß es zukünftig Probleme geben wird, wenn z.B. ein kleinerer Newcomer Strom durch das Netz eines oder gar mehrerer Netzbetreiber-Elektrizitätsversorgungsunternehmen (EVU) leiten will. Im Rahmen einer freiwilligen Vereinbarung zwischen dem Bundesverband der Deutschen Industrie e.V., dem Verband der Industriellen Energieund Kraftwirtschaft und der Vereinigung Deutscher Elektrizitätswerke (VDEW), der sogenannten Verbändevereinbarung, werden Kriterien und Preise für den Netzzugang und die Bestimmung der Durchleitungsentgelte vorgeschlagen. Diese Vereinbarung ist für die Energieversorgungsunternehmen zwar nicht verbindlich, wird jedoch in Zukunft in den meisten Fällen für die Bestimmung des Durchleitungsentgelts herangezogen werden. Problematisch hierbei ist, daß die vorgesehene Regelung der im Energiewirtschaftsgesetz vorgeschriebenen Kostenorientierung nicht gerecht wird. Vielmehr wirkt die ungerechtfertigte Kostenzuweisung für bestimmte Durchleiter diskriminierend und führt zu Gewinnmitnahmen bei den netzbesitzenden Energieversorgungsunternehmen. Es ist sogar strittig, ob die Vereinbarung wettbewerbsverzerrend, und deshalb kartellrechtlich zulässig ist.

Es besteht zwar die Möglichkeit zum Erlaß einer Rechtsverordnung, die die Bestimmung transparenter und nichtdiskriminierender Durchleitungsbedingungen und -entgelte regeln könnte, aber sowohl Bundesregierung als auch Bundesratsmehrheit bauen bisher auf den Erfolg der Verbändevereinbarung.
Problematisch sind vor allem Regelungen, die die Durchleitung von Strom teuer machen bzw. erschweren. Die Durchschnittsnetzkosten enthalten Investitionen bzw. Abschreibungen des Kapitals, Wartung, Instandhaltung sowie ein Gewinnaufschlag. Diese Kosten fallen allerdings real bei der Durchleitung nicht an. Besonders bei der Durchleitung von Leistungen mit geringen Jahresbenutzungsstunden übersteigen die Durchleitungsgebühren die Erzeugungskosten im Kraftwerk teilweise um ein Mehrfaches.

Durchleitungsgebühren sollten jedoch für alle Akteure gleich, transparent sowie einfach und schnell handhabbar sein, um möglichst viele Wettbewerber in den Wettbewerb einbeziehen zu können. Zusätzlich sollten sie für die Netzbetreiber ökonomische Anreize sichern, damit sie trotz des natürlichen Monopols und der praktizierten Durchschnittskosten-Preisbildung an einer Effizienzverbesserung interessiert sind. In Großbritannien hat sich das sogenannte ,pricecap“-Prinzip, eine (obere) Begrenzung der Preisentwicklung, bewährt (2).

\section{Regenerative diskriminiert}

Die Durchleiter müssen für die Netzbenutzung für jeden Netzbereich und jede Netzebene Leistungskosten bezahlen. Dabei richtet sich das Entgelt an der höchsten Leistung ( der höchsten Strommenge bezogen auf die Zeiteinheit) aus, die der Durchleiter im Laufe eines Jahres mit seinem Abnehmer vereinbart bzw. liefert. Diese Regelung ist insbesondere für regenerative Energiequellen ungünstig, weil diese nur eine beschränkte Ausnutzungsdauer ihrer installierten Leistung pro Jahr haben. Es kann gezeigt werden, daß aufgrund der leistungsbezogenen Ermittlung der Durchleitungsgebühr etwa der dreifache Wert der tatsächlich im Durchschnitt anfallenden Netzkosten berechnet wird. 
Die heutige Abrechnungspraxis der Energieversorgungsunternehmen ist ein Beleg dafür, daß keine Notwendigkeit für eine solche Regelung besteht: die Haushaltskunden werden nicht nach ihrer maximalen Bezugsleistung abgerechnet sondern nach den bezogenen Jahresverbrauch.

Die Verbändevereinbarung sieht bezüglich Einspeisung und Entnahme eine Zeitgleichheit vor, wobei allerdings Details, wie streng diese Gleichzeitigkeit $\mathrm{zu}$ handhaben ist, noch nicht vorliegen. Eine gleichzeitige Lieferung und Abnahme der Strommengen würde für einen unabhängigen Stromerzeuger, der Solarstrom an die Haushalte liefern möchte, folgendes bedeuten:

- das Unternehmen kann nur dann liefern, wenn die Sonne scheint, oder

das Unternehmen kann keinen reinen Solarstrom anbieten und muß dementsprechend einen Mix aus regenerativen Energiequellen oder einen Mix aus regenerativen und fossilen Energiequellen anbieten.

Diese Regelung behindert insbesondere das Angebot von regenerativem Strom.

\section{Ein Beispiel}

Das folgende Beispiel verdeutlicht die derzeitigen Schwierigkeiten, wenn ein Kunde von seinem Wahlrecht Gebrauch machen möchte (3): Dr. Wolfgang Roos, im Öko-Institut Freiburg für den Bereich Energie zuständig, beantragte bei der Freiburger Energie- und Wasserversorgungs AG (FEW) den Strombedarf seines Hauses in Höhe von 350 Kilowattstunden soweit als möglich durch ein rapsölbetriebenes Blockheizkraftwerk durchzuleiten. Nach seinen Berechnung betragen die Stromverteilungskosten der FEW etwa $4 \mathrm{Pf} / \mathrm{kWh}$. Hinzu kommen Konzessionsabgaben in Höhe von 3,91 Pf/kWh. Die FEW ist verpflichtet, zu den allgemeinen Versorgungsbedingungen und Tarifen mit Zusatz- und Reservestrom zu versorgen. Die FEW unterbreitete daraufhin ein Angebot bestehend aus einer einmaligen Antragsgebühr zur Abdeckung der zusätzlichen Verwaltungskosten sowie aus einer jährlichen Pauschale, die sich an der Abnahmemenge orientiert. Über die Höhe der Angebotskomponente war man bereit zu verhandeln.

Herr Roos widersprach, indem er sich auf die gesetzliche Verpflichtung der Energieversorger die Durchleitung zum kostendeckenden Entgelt durchzuführen sowie dieses zu benennen, berief. Es sei nicht zulässig, daß der vor dem Hintergrund des neuen Energierechts entstehende Personalaufwand in Rechnung gestellt würde.
Zur Zahlung einer Verrechnungspauschale für den zusätzlichen Abrechnungsaufwand von 10 Mark erklärte er sich bereit.

Die FEW unterbreitete daraufhin ein Angebot für abrechnungstechnische Tätigkeiten sowie eine Konzessionsabgabe in Höhe von 66 Mark pro Jahr ohne dieses weiter aufzuschlüsseln. Dies liegt etwa das doppelte über ihren Kosten. Zudem wurde der Forderung nach Transparenz nicht entsprochen.

\section{Fazit}

Die Mehrzahl der potentiellen Stromerzeuger oder -anbieter bzw. Stromabnehmer erhalten durch die Verbändevereinbarung keine wettbewerbsfähigen Stromdurchleitungsbedingungen (4). Viele Kunden müßten für die Durchleitung mehr bezahlen, als ein mittlerer Industrieverbraucher für die Erzeugung, den Transport, die Verteilung und die Reservehaltung in der Summe bezahlt. Neue Anbieter wären bei ungeklärten Durchleitungsbedingungen nicht bereit,

Investitionen $\mathrm{zu}$ tätigen.

Überall im Ausland, wo der Strommarkt bereits liberalisiert wurde, ist der Netzzugang und die Netznutzung nicht im Rahmen einer Vereinbarung der interessierten Verbände sondern staatlich geregelt (Skandinavien, England, Niederlande, USA). Hier basieren die Durchleitungsentgelte nicht auf Leistungspreisen sondern überwiegend auf strommengenabhängigen und entfernungsunabhängigen Tarifen. Dies eröffnet kleineren Stromanbietern und -abnehmern sowie Strom aus regenerativen Energiequellen faire Marktchancen.

\section{Anmerkungen}

(1) Siehe $\S 6$ ff. EnWG (Gesetz zur Neuregelung des Energiewirtschaftsrechts).

(2) Zu diesem „price-cap"-Prinzip siehe Weisheimer, Martin: Wie könnte dem fehlenden Wettbewerbsdruck bei der Stromdurchleitung begegnet werden? in: Wirtschaft im Wandel 6/98, Institut für Wirtschaftsforschung, Halle.

(3) Zum Briefwechsel zwischen Herrn Dr. Roos und der FEW siehe Energie Depesche 3/98, S.14 - 15.

(4) Siehe hierzu ausführlicher die Studie: Das neue Energierecht - Probleme der Stromdurchleitung vom Öko-Institut Freiburg.

\section{Der Autor}

Dietmar Graf ist seit 1998 als Redakteur beim ökom Verlag tätig

Kontakt: ökom Gesellschaft für politische Kommunikation mbH, Waltherstr. 29, 80337 München,

Tel. $089 / 544184-60$, Fax -99

\begin{abstract}
Die anstiftung ist eine gemeinnützige Forschungsgesellschaft, die zukunftsweisende Projekte im sozialen, kulturellen und ökonomisch-ökologischen Bereich durchführt.
\end{abstract}

Für unser interdisziplinäres und praxisorientiertes Team suchen wir ab sofort

\section{den/die ReferentIn für Öffentlichkeitsarbeit (30 Std./unbefristet)}

Sie haben berufliche Erfahrung mit Öffentlichkeitsarbeit im sozialen oder ökonomisch-ökologischen Bereich und Lust darauf, sich für den erfolgreichen Auftritt einer zukunftsorientierten Forschungsgesellschaft einzusetzen. Sie schreiben routiniert, sind sprachlich gewandt und haben ein sicheres Gefühl für Gestaltung. Wir benötigen Ihre Mitarbeit bei unseren mündlichen, schriftlichen und elektronischen Präsentationen.

\section{Ebenfalls ab sofort \\ eine/n jüngere/n Sozialwissenschaftlerln (mind. 32 Std./unbefristet)}

Ihre Aufgabe ist es, für verschiedene Forschungsprojekte der anstiftung Grundlagen zu erarbeiten und Projektkonzeptionen wissenschaftlich zu unterstützen. Sie sind vertraut mit aktuellen sozialpolitischen und ökonomischökologischen Fragen, recherchieren und schreiben gern.

Weiterhin ab sofort

\section{eine/n Mitarbeiterın für die Projektberatung} (mind. 32 Std./befristet bis August 2001)

Sie wirken daran mit, erprobte Ideen der anstiftung (v.a. Eigenarbeit) in anderen Orten zu verbreiten. Sie haben Projekterfahrung, können Menschen begeistern und beherrschen Dokumentation und Informationsmanagement.

Die Stellen werden in Anlehnung an BAT vergütet.

Wir freuen uns auf Ihre schriftliche Bewerbung bis 14.03.1999.

\section{anstiftung ggmbh Daiserstrasse 15, Rgb. 81371 München}

Weitere Informationen erhalten Sie unter 089 - 7474 60-0 und im Internet unter http://www. anstiftung.arg.net 
(c) 20I0 Authors; licensee IÖW and oekom verlag. This is an article distributed under the terms of the Creative Commons Attribution Non-Commercial No Derivates License (http://creativecommons.org/licenses/by-nc-nd/3.o/), which permits unrestricted use, distribution, and reproduction in any medium, provided the original work is properly cited. 